\title{
Smart Manufacturing and Jobs
}

\author{
Juvenal Mendoza-Valencia ${ }^{1}$ \\ Instituto Politécnico Nacional, México
}

\begin{abstract}
The proper use of new technologies, together with a good management of these by the workers, makes the workplace become a melting pot of new ideas, where professional development and motivation [1] make a difference with past and present times where monotonous and repetitive work was the constant. With the use of artificial intelligence, the cloud and big data, a set of intelligent sensors is necessary, which can monitor a critical variable within the production line, which requires an encrypted communication infrastructure and with information that can be monitored in real time, which allows a better performance of the company, making it a more pleasant place for staff and with working conditions that help to overcome them when performing complex tasks. Due to the educational level that many of the workers currently have and the fact of being born in a technified society, it causes them to be more interested in carrying out activities where they develop their creativity and skills, resulting in economic and psychological well-being.
\end{abstract}

Keywords. Manufacturing, worker and artificial intelligence

\section{Introduction}

The companies that take advantage of new technological developments in a creative way are those that remain in the market; for this they must promote among their staff the quality of having critical thinking, problem solving, analytical reasoning and collaborative work [2], for this it is necessary that the organization has a horizontal structure to be democratic, in this way they can have the conditions to adapt to change.

If we change our business approach from short term to long term, we can be in line with technological development, it follows a series of steps which range from: development, growth, maturity and obsolescence, in this way we can seek the staff of the different positions depending on the activity carried out table 1.

Table 1.- Job relationship vs technology development curve

\begin{tabular}{|c|c|}
\hline Market Stall & Technological level \\
\hline Direction & Onward development \\
\hline Management & Growth forward \\
\hline Technical & Maturity \\
\hline Operative & Maturity \\
\hline
\end{tabular}

Source: self made.

If we consider the importance of table 1, we can look for personnel in the different areas where the greatest emphasis of hiring is the interest of future 
knowledge of new technologies, so that you can relate what is current with future trends, which it will allow you to anticipate changes.

\section{Method}

For the following research, a field study was carried out in SMEs located in the metropolitan area of Mexico City, the purpose was to analyze their organizational culture, the degree of motivation of their workers and commitment of management and direction in this regard; For this purpose, interviews were conducted with the personnel of the three hierarchical levels. Likewise, a bibliographic search was carried out that covered topics of motivation in employment and technologies of industry 4.0, with the aim of analyzing their possible impacts on the factories of the future.

\section{Workers past, present and future}

When the industrial revolution began at the end of the 18th century, the vast majority of the population belonged to the agricultural sector, so knowledge revolved around it, the concern centered on famines, diseases and wars [3], At the beginning of the 20th century, industrial processes are consolidated and mass production is born, where workers are specialized in an activity within it, which is accompanied by an increase in goods, the uncertainty about what will happen in the future decreases, for what is more confident, being conducive to the functioning of the economy in general.

In the twentieth century, the population became more urban and only $2 \%$ were in agricultural activities [3], those born in it seek to develop a technical skill, to be able to insert themselves in industry, those who achieve it are guaranteed well-being, this is known as a welfare state policy [4].

The rhythm of work is determined by the market, so there is a duality between the possibilities of the factory and its demands. For this purpose, we have to look for indicators that allow us to know how its behavior is; This is reflected in an increase or decrease in the speed of the production chain; so the worker must be willing to bear the stress that it causes.

It is said that in the industrial world nothing is more constant than change, which means that there are times where there is a lot of work and others where it is scarce, which causes a cycle of hiring and firing, the worker must strive to be of the essential part of the production line, so that it has a permanence in the time and in this way reduce its economic uncertainty.

Over time, new technological developments appear, the requirements that they demand, make the skills of the workers have to change, from there the speed of change will be determined, which allows establishing training cycles, start-up and execution of them, This is where the difference between avant-garde companies and followers is achieved, the former develop revolutionary ideas, socialize them and set the standard for price, quality and variety; causing the latter to be forced to adopt said changes.

The process of socialization of the implementation of new technologies is a slow process, which implies the search for the worker's profile, many hours of training and 
understanding of the potential of technology in the field of production; Many times workers, technicians and engineers do not understand their purpose, so at some time they work with very little vision of what really happens.

Leading companies will have enough time to put their ideas into practice on how to use new technologies and train staff to do so; on the other hand, those that do not do so will work with a lot of stress when they implement these changes, due to the fact that they have new parameters that mark the advances in the world of technology.

Many psychologists and sociologists have generated the idea that individuals fear change, being the reason why technology takes time to be exploited in factories; more recent studies indicate that this is true with certain generations like the baby boomer and $\mathrm{X}$; but the millennials and the $\mathrm{Z}$ look for new experiences that break the monotony of daily work [4].

In recent studies, it is said that only $15 \%$ of workers are committed to their tasks [4], we have a big problem to motivate the workforce, so it is natural that companies prefer less developed countries to install their factories; But when their population becomes urban and therefore industrial, they will have productivity problems again, because future workers are no longer interested in the positions that are offered.

We must bear in mind that a production line has a normal statistical distribution in the demand for its products, the reason is due to the different seasons of the year, which means that $80 \%$ of production is concentrated in some models and $20 \%$ corresponds to the rest, this means that $80 \%$ of the time will be practically doing the same work and $20 \%$ will be doing different things (fig. 1), so the recruitment of new talented workers will be more and more problematic [5].

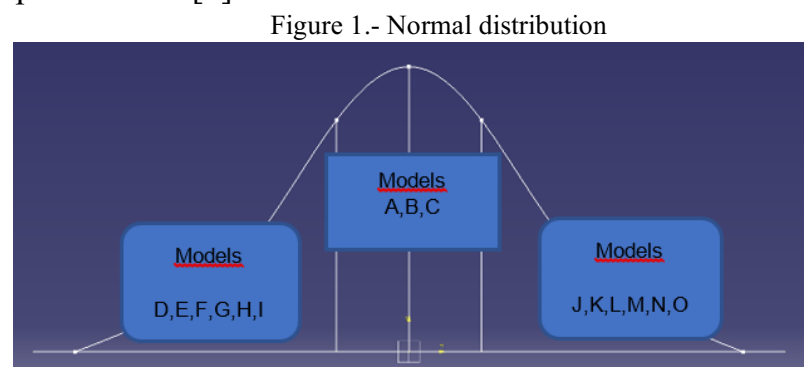

Source: self made.

If we take into account that every day, as a result of the processes of globalization, production volumes tend to increase by millions of units, this means that routine and repetitive operations will be practically all the time, so that workers, technicians and engineers will be subjected to great stress, to be able to stay in the workplace and if not, the only solution is to leave it, which explains why staff turnover is high $12 \%$ annually.

Hence the importance of making a good analysis of the production line, to determine with certainty which areas are dangerous, with a lot of physical effort, monotonous and that demand very little creativity; having this information, it is possible to plan the increase in capacity of the line demanded by the market, the intelligent technology that supports it and the profile of the worker that will keep it running.

Once it is decided to implement intelligent manufacturing in the lines, the human resources department can begin to study the jobs, looking in historical data for the 
following points: worker interest in the activity, education demanded by the position, stress, creativity required and innovation in problem solving; when the results are obtained, a comparison is made with the percentage of rotation that was had before using the smart equipment: if it is greater, equal or less; consequently determining if their expectations are met.

In this way, we can influence the study programs in high school and universities, emphasizing the fields of interest shown by the personnel in the industrial area, with which the future profile of the students can be adjusted to the trends of the intelligent manufacturing, the purpose is to ensure that the resources invested in education are increased to have better workers, engineers and entrepreneurs, who can be leaders in the industrial branch.

\section{Artificial intelligence and employment}

If we start from the fact that all technological progress has as its primary objective the enhancement of human capabilities, which will allow the benefits of Science and Technology to spread in society and that it will determine the ways in which it will use it in its daily life, thus generating general well-being.

In order for artificial intelligence to take place, it is necessary that there be a communications infrastructure inside the factory, which allows wired and wireless communication, it is also very important to establish the technological structure by levels: operational, field, control, managerial and business [6], which will generate a set of data, for this purpose we will require a genetic control algorithm to transform them into different phases: 1st. an analysis, 2nd. in information, 3rd. knowledge, 4th. control and 5th improvement [7], each time an iteration occurs, the system will make small modifications to process them in the genetic algorithm, producing an evolution in decision-making over time [8].

If the man-made objects can be related to the environment, transfer the data to the cloud, analyze it, determine a better way to execute the work with the use of genetic algorithms and make a transfer to the emitting object; will result in better performance, over time all our devices will be operating optimally [9].

As there are millions of objects operating in the greatest diversity of places, environments, conditions and people's needs, in the cloud together with their analytics, they will be able to make probabilistic models such as: control charts, Kpi, Cpk, mean time between failures and maintenance 4.0; In order to take advantage of the wealth of massive information, Big data will be used [10], the results will be adjusted to reality, give recommendations for use, maintenance and replacement, with which the confidence of the production line will tend to $100 \%$.

If we have already defined what we are going to do with the data generated by intelligent machines and the evolution in time that it entails, it cannot be said that up to this point the task is already completed, the human being performs: a geographical mobility, he has social relations, has his own point of view, understands public and business policies, market needs and understands his social environment; which are changing dynamically, that is, it is a unique being in the world and therefore has an 
infinite wealth of information, which it would be necessary to consider to reduce the duality between what the factory offers and what the market demands.

For this purpose it is necessary for the company to develop a network intelligence to have the information required by each of the members, with the aim of communicating in real time, to know what are: the activities to be developed, the incidents, the models to be produce, volume, cancellations, etc .; These computer programs must be executed on $6 \mathrm{G}$ smartphones in order to meet the communication demands, which must run at speeds of $1 \times 10^{\wedge}(-9) \mathrm{s}[11]$, which allows supporting internal and external requirements of the IIoT.

As the work of data collection, analysis and execution will be in charge of intelligent machines, the human environment is focused on the development of innovation and collaborative work; For this purpose, the members of the areas of the company must be mature enough to leave behind the problems that arise in current companies such as: envy, hatred, vanity and pride; which do not allow the group to work together; those in charge of production have to be solving these rather than looking to the future.

Smart work centers will be more concerned with analyzing the psychological conditions of their members in order for their cooperative spirit to emerge and in this way generate innovative developments whose purpose is an original point of view, a product of the culture of their members. participants.

If we rely on artificial intelligence, as a way to put these ideas into practice on the production line, we will therefore have original products that attract the attention of the consumer, due to the practicality of their ideas, ease of maintenance and solutions to problems. specific that are presented at a given time, so that the buyer can be satisfied with the property purchased.

The new technologies of the 4th industrial revolution such as: artificial intelligence, big data and the cloud; They must allow us the emergence of new relations of production, where the human spirit will set the standard [12] if the purpose is to ensure that all internal and external participants in the production chain have the common benefit as their objective, it will be possible to leave behind practices such as the search for places with low wages; By changing our purpose, the markets where the plants settle will develop, creating new consumers.

With an increase in demand, the work of producing the goods is left to the intelligent system; Therefore, the workers will focus on solving fundamental problems such as: level of flexibility of the plants, ecological impact, recycling, caring for the environment, assigning the good to the customer at the time he makes the purchase, working with the social part, benefits and damages that our product may have, governmental aspects, future trends [13].

When we have a work group that relies on artificial intelligence, boring, repetitive, monotonous and dangerous jobs are left to machines [14]; Therefore, the culture that each of its members has becomes very valuable, due to the fact that it defines the ways of being of the people where they are: their dreams, joys, their fears, anguish, hopes, longings, etc. where the pursuit of happiness is the reason for their lives.

The word work will change its meaning to self-realization, own identity, cultural expression of the group and melting pot of new ideas, so that workers will be more identified with the company and their commitment in a percentage of 80 to $90 \%$ and the 
staff turnover of 2 to $3 \%$, this should be reflected in an increase in productivity, quality and variety.

Currently it is said that $84 \%$ of the value of a company is represented by the intangible part and $16 \%$ by the tangible; Until now, the creation of innovative products has been carried out outside the factories, so the people involved in their development lack knowledge of how this is produced, so the result is products that do not respect the life of the worker they are polluting and when their life cycle ends, it becomes an industrial waste problem, so it is common to see them anywhere.

We must seek that in future smart factories there is no such dualism and that creators and innovators have direct contact with smart manufacturing and in this way seek to solve the problems that arise at the time of good manufacturing, transportation, marketing, consumption and recycling; with which a new sustainable economy can be generated, reflected in cleaner environments for the future [15].

If the innovators work hand in hand with the workers, they will provide mutual feedback and $100 \%$ ecological products will come out throughout their distribution chain, artificial intelligence will be able to support activities with a lot of stress due to a punctual increase in demand and will find solutions that help to complex decision making, with multiple variables and short response time.

We need quality jobs, with complex activities, the creation of new consumers, the distribution of wealth to guarantee social stability and unrestricted respect for the dignity of the person, all of which will translate into a new social approach, which makes people breathe in to more challenges big.

\section{Results}

Companies have a short-term focus, which limits them to prepare for the future, for this purpose they seek to have a competitive advantage in the area in order to carry out the work they carry out on a daily basis and in this way they hope to stay in the market; which sometimes depends on their geographical location, the service that the product demands, the opportunity time, cost of the good and variety, so if they take advantage of them, their permanence can result.

With regard to the worker, the managerial part is the one that is most aware of the importance of permanence, promotion, development and organizational culture; Therefore, they establish strategies to achieve said purposes; but if for the achievement of these it is necessary to disburse additional amounts of money to which it is normally destined, the management is strongly opposed, they consider it an expense, due to the fact that at any time they can go and lose the investment.

\section{Discussion}

Workers are being influenced every day by the appearance and use of new intelligent technologies, they allow them to relate horizontally and vertically in the company, having by the mere fact of doing so, a combinatorial power for solving problems to 
almost unlimited real time; Not wanting to recognize this new reality leaves us at a disadvantage to those companies that do.

For the use of new technologies, what has to be considered is the degree of maturity of these, in this way the real potential is known, with which the possible application can be defined with certainty; Likewise, it is more attractive for the worker to develop an activity within the factory that gives him the opportunity to grow as a person, interact and develop his full potential.

\section{Conclusion}

We are in the 4th industrial revolution where new technologies are appearing, the most important for the manufacturing area is Artificial Intelligence, for this purpose we must define what we want from this set of new technological advances, in order not to repeat the error that it was committed in the 3rd industrial revolution where we only focused on putting it into practice, forgetting that it should serve the human being; so after 40 years we have problems of: distribution of wealth, ecological, health, social, etc .; at this moment we have in our hands the power to make a change where all participants benefit.

We also have to consider that once the guidelines that will mark said transformation are established, a dynamic that supports them is generated and over time it only grows, so it is impossible to make any change once it is underway.

It is worth noting the fact that, in the history of industrial revolutions, when the human being is not at the center (1st and 3rd), technological advance only benefits some people who have a close relationship with the centers of power and When the human being is the main objective, there is a benefit for society, as is the case of the 2 nd industrial revolution and that could be the case of the 4 th.

\section{Acknowledgements}

To the National Polytechnic Institute and the UPIICSA Postgraduate Studies Section and especially to the Master of Industrial Engineering, for all the support received for the preparation of this article.

\section{References}

[1] Agrawal A., Gans J. Goldfarb A Prediction machines, $1^{\text {a }}$ ed. USA: Harvard Business Review; 2018.

[2] Brynjolfsson E., Mcafee A. The second machine age, $1^{\text {a }}$ ed. USA.: W. W. Norton and company, Ing; 2014.

[3] Noah Y. Homo Deus, $1^{\mathrm{a}}$ ed. México: Debate; 2019.

[4] Clifton J. Harter J. It's the manager moving from boss to coach, $1^{\mathrm{a}}$ ed. USA: Gallup press.

[5] Chopra S., Meindl P. Supply Chain Management, 5ª ed. USA: Pearson; 2013.

[6] Veneri G., Capasso A. Hands-on Industrial Internet of things, $1^{\text {a }}$ ed. UK: ed. Packt; 2018.

[7] Pérez M. Big Data, $1^{\text {a }}$ ed. México: Alfaomega; 2016.

[8] García A. Inteligencia Artificial, $1^{\text {a }}$ ed. México: ed. Alfaomega; 2017. 
[9] Caiza G., Saeteros M., Oñate W., Garcia M. Fog computing at industrial level, architecture, latency, energy, and segurity, ELSEVIER 2020: Heliyon 6 (2020) e03706.

[10] Ponce P. Inteligencia Artificial, 25ª ed México: Alfaomega; 2019.

[11] Zhou Y., Liu L., Wang L., Hui N. Service aware 6G: an intelligence and open network base don convergence of communication, computing and caching. ELSERVER 2020 May; B.V. on behalf of KeAi Communications Co. Ltd..

[12] Phuyal S., Bista D., Bista R. Challenges, opportunities and future directions of Smart manufacturing. ELSERVER 2020 sustainable Futures 2 (2020) 100023.

[13] Beier G., Ullrich A., Niehoff S., Reibig M., Habich M., Industry 4.0: How it is defined from a sociotechnical perspective anda haw munch sustainability it includes. ELSERVER 2020 Jornal of cleaner Production 259 (2020) 120856.

[14] Sgorbossa F., Grosse E., Neumann W. Human factors in producction and logistics systems of the future. ELSERVER 2020 Anual Reviews in control 49 (2020) 295-305.

[15] Knapcikova L., Balog M. Industry 4.0: Trends in management of intelligent manufacturing System, $1^{\mathrm{a}}$ ed. Switzerland: Springer:2019. 